Www.jmscr.igmpublication.org

Impact Factor (SJIF): 6.379

Index Copernicus Value: 79.54

ISSN (e)-2347-176x ISSN (p) 2455-0450

crossrefDOI: https://dx.doi.org/10.18535/jmscr/v6i12.84

Journal Of Medical Science And Clinical Research

IGM Publication

An Official Publication of IGM Publication

\title{
Granulomatosis with Angitis of Lung treated with Steroids and Rituximab- Case Report and Review of Literature
}

\author{
Authors \\ Dr Jolsana Augustine ${ }^{1}$, Dr Rajesh $\mathbf{V}^{1^{*}}$, Dr Latha Abraham², Dr Dantis Immanuel ${ }^{3}$ \\ ${ }^{1}$ Department of Pulmonary Medicine, ${ }^{2}$ Department of Pathology, ${ }^{3}$ Department of Immnunology and \\ Rheumatolgy, Rajagiri Hospital, Aluva, India
}

*Corresponding Author

Dr Rajesh V

Consultant Pulmonologist, Department of Pulmonary Medicine, Rajagiri Hospital, Aluva, Kerala, 683112

Email: jolsana.augustine@ rajagirihospital.com, Mobile number: 9446728953, 0484- 29055039

Abstract
Granulomatosis with angitis, formerly termed Wegener's granulomatosis (WG) is an uncommon
autoimmune disease with multi-system involvement, the pathological hallmark of which is necrotizing
granulomas with vasculitis. Although it commonly limits itself to involvement of the upper and lower
respiratory tracts or kidneys, it may potentially involve any organ. We present the case of a young lady
who presented with persistent fever lasting 2 months and right upper lobe consolidation, with laboratory
evidence of glomerulonephritis. Serological tests revealed high titres of antibody to proteinase 3
(antineutrophil cytoplasmic antibody - cytoplasmic type-ANCA). A lung biopsy from the left upper lobe
revealed necrotizing granulomas with vasculitis, thus clinching the diagnosis. Remission was achieved
with a combination of prednisolone with rituximab, complete remission being evidenced as early as 3
weeks of therapy initiation. Steroids were gradually tapered in the maintenance phase and she remains
under close follow up for any features of relapse.
Keywords: Granulomatosis with angitis, necrotizing granulomas, small vessel vasculitis, Wegener's
granulomatosis.

\section{Introduction}

Wegener's granulomatosis has been classically described as a systemic vasculitis (polyangiitis) with a triad of necrotizing granulomatous inflammation of the upper and lower respiratory tracts, systemic necrotizing vasculitis, and glomerulonephritis. ${ }^{(1)}$ Consensus statements from various international societies have recommended that the name Wegener's granulomatosis be changed to granulomatosis with polyangitis abbreviated as GPA. ${ }^{(2)}$ Most of the affected patients have either upper airway or pulmonary involvement, and many subjects have both. Nonspecific complaints of fever, anorexia, weight loss, and malaise are often present. The radiological findings may vary from cavitating parenchymal nodules to features of alveolar haemorrhage. Serological testing for ANCA and tissue biopsy from the affected site (demonstrating necrotising granulomatous inflammation with vasculitis) clinches the diagnosis in most situations. The disease has a progressive course 
with mortality reaching upto $90 \%$ at 2 years if left untreated and immunosuppression holds the key in achieving and maintaining remission.

The present report narrates a young lady who presented with persistent fever and left upper lobe consolidation in chest radiograph, who on evaluation had features of active glomerulonephritis. Serological testing for ANCA and a CT guided trucutlung biopsy established the diagnosis of GPA. Remission was achieved with a combination of prednisolone with rituximab within 3 weeks of therapy initiation.

\section{Case Summary}

A 23 year old lady, software engineer by profession, with no comorbid illnesses, presented to the pulmonary medicine out-patient department with intermittent high grade fever for 2 months. She denied any background of atopy, occupational exposures, tobacco product use or high risk sexual behavior. There was no associated cough, shortness of breath or chest pain. Bowel and bladder habits were normal. There was no loss of appetite or weight loss. Symptoms referable to the upper respiratory tract were absent, as was any features of skin or joint involvement. Physical evaluation revealed normal vital signs except a temperature of $100.8 \mathrm{~F}$ and proportionate tachycardia. Skin, joints and neck examination was unremarkable.No palpable peripheral lymphadenopathy was noted. Respiratory evaluation revealed crackles in left upper chest. Examination of other systems was unrewarding. Chest radiograph revealed left upper lobe alveolar densities (Fig 1), which was corroborated in high resolution CT chest. No evidence of pleural involvement, airway lesions or mediastinal nodes was made out in CT chest. Sputum examination for gram stain and culture, as well as acid fast bacilli was negative. Serology for autoimmune diseases (rheumatoid factor, ANA and ANCA) were requested. Multiple blood cultures returned negative. Urinalysis showed 60-70 RBCs / hpf. A fibreoptic bronchoscopy revealed non-inflamed airways; BAL for bacterial and fungal cultures as well as mycobacteria by gene Xpertwas unrewarding. A CT guided biopsy from the consolidated left upper lobe revealed necrotizing granulomatous inflammation with vasculitis (Fig 2,3). The anti PR3 levels, which were available by this time, revealed grossly elevated titres. A diagnosis of GPA with pulmonary and renal involvement was thus arrived at.

After updating the patient and family about the diagnosis, potential clinical course and therapeutic options, an induction regimen was initiated with 1 gram of methyl prednisolone for 3 consecutive days followed by a dose of 1 gram of rituximab. An oral steroid at a dose of $60 \mathrm{mg}$ of prednisolone was initiated from fourth day. The temperature touched baseline by the third day and chest radiograph showed early features of resolution by the $5^{\text {th }}$ day of initiation of induction regimen. A second dose of rituximab was given 2 weeks later. Evidence of glomerulonephritis in urinalysis disappeared by 2 weeks and the chest radiograph by this time revealed total resolution (Fig 4). Steroids were gradually tapered in the maintenance phase and she remains under follow up for any features of relapse.

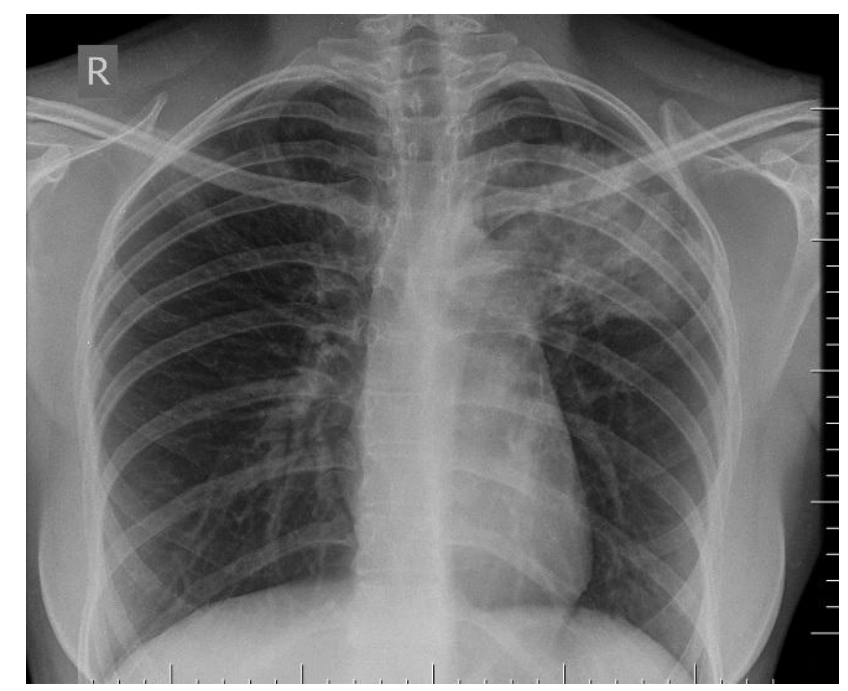

Figure 1 Chest radiograph showing left upper zone consolidation 


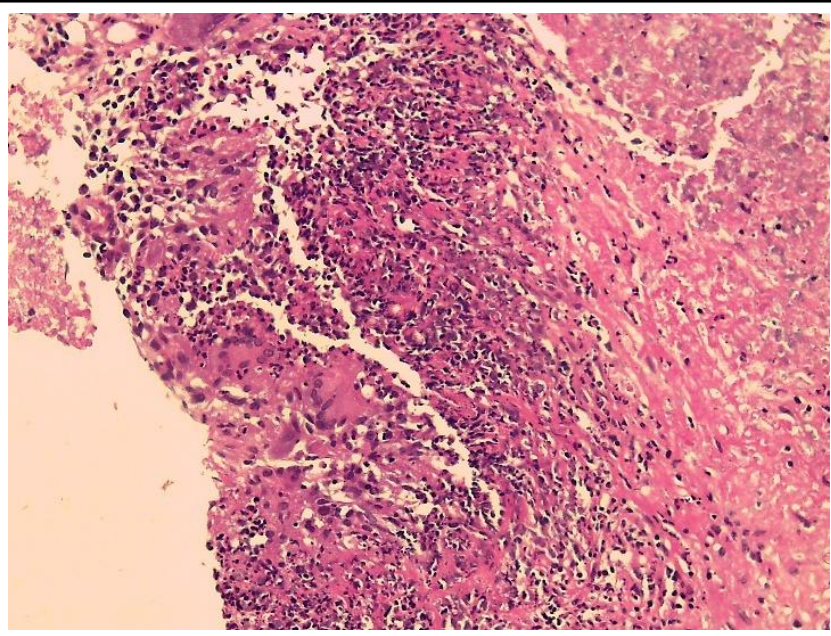

Figure $2 \mathrm{H}$ \& E, 100x photographs demonstrating necrotising granuloma with multinucleated giant cells and palisading histiocytes

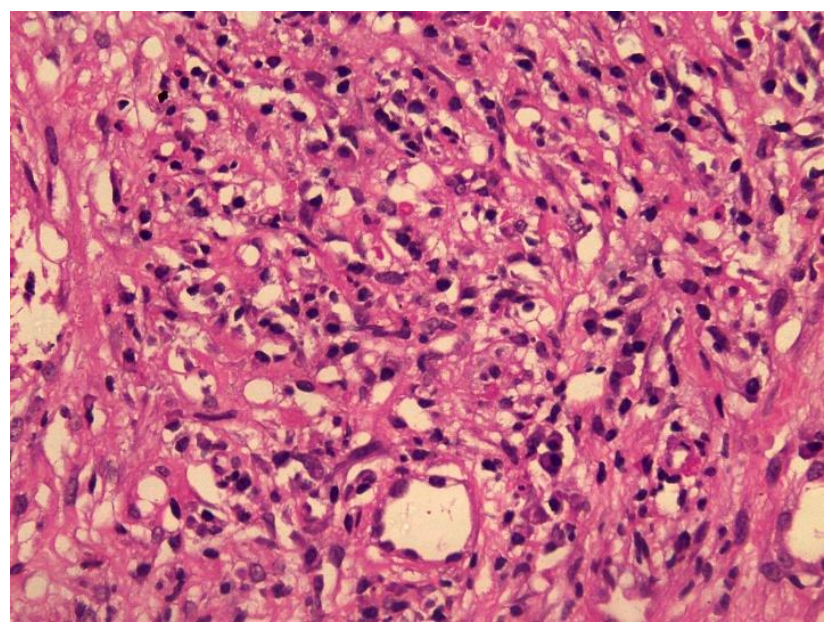

Figure 3 H\& E, 400x showing destructive angitis

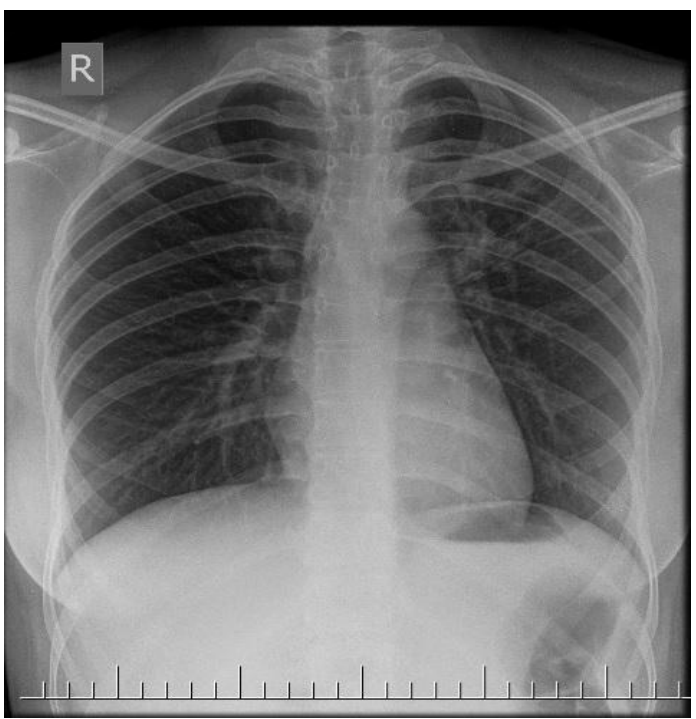

Figure 4 post treatment image demonstrating reasonable clearance of left upper zone opacity

\section{Discussion}

Granulomatosis with polyangiitis is an uncommon disease. Although most commonly affecting the respiratory and renal systems, any organ or system may be involved since the disease is a systemic vasculitis. The spectrum and severity of symptoms in pulmonary disease vary considerably and onethird of patients can be asymptomatic. At the other end of the spectrum, acute and fulminant alveolar haemorrhage with respiratory failure can ensue.

The commonest radiological abnormality is lung parenchymal nodules, many of which tend to cavitate. $^{(3)}$

In ANCA-associated vasculitis (AAV), B-cell activation and levels of B-cell-activating factor correlate with disease activity. ${ }^{(4)}$ Serological testing for c-ANCA (antineutrophil cytoplasmic antibody) is crucial for establishing the diagnosis of GPA and other ANCA associated vasculitis, indirect immunofluorescent assay being the preferred mode of testing. More than 90 percent of patients with active GPA (with both lung and kidney involvement) or microscopic polyangiitis have demonstrable titres of ANCA in the serum. However, in limited forms of GPA, ANCA testing may be negative. The majority of patients with GPA have autoantibodies directed against serine proteinase 3 (PR3), known as PR3-ANCA (CANCA), that are associated with diffuse cytoplasmic staining. ${ }^{(5)}$

Tissue confirmation of GPA is highly desirable, even among patients with a compatible clinical presentation and positive serology, due to the potential toxicity of treatment. The choice of a biopsy site must be individualized based on the accessibility of specific sites of disease and risk of procedure. Lung biopsy is preferred for the evaluation of single or multiple pulmonary nodules, particularly when extra-pulmonary disease is absent or not accessible to biopsy. Wegener's granulomas contain CD20-positive, Bcell-rich, follicle-like areas with affinity for the proteinase 3 ANCA antigen. ${ }^{(4)}$ Even when GPA is strongly suspected (positive ANCA), infection and neoplastic disease affecting the lung must be 
excluded prior to initiating immunosuppressive therapy.

Therapy for GPA has two main components: induction of remission with initial, rather intense immunosuppressive therapy, and maintenance of remission with slightly weaker immunosuppressive therapy for a variable period to prevent relapse. The initial choice of agents depends on the severity of disease at presentation. A variety of methods have been used to assess disease activity in patients with granulomatosis with polyangiitis (GPA). The Birmingham Vasculitis Activity Score (BVAS) $)^{(6)}$ has been extensively employed in patients with either GPA or MPA, specially so in the research settings. The initial immunosuppressive therapy in GPA typically employs steroids with a second agent, either cyclophosphamide or rituximab. ${ }^{(7)}$ This regimen is particularly suited for subjects with organ threatening or life threatening disease (active glomerulonephritis, pulmonary hemorrhage, cerebral vasculitis, progressive neuropathy, orbital pseudotumor, gastrointestinal bleeding, pericarditis, or myocarditis). Rituximab is considerably less toxic than cyclophosphamide, although an expensive agent.

Two randomized trials have suggested that rituximab is an effective alternative to cyclophosphamide for the initial treatment of patients with newly diagnosed GPA. The rates of serious adverse events were similar with both drugs. The RAVE trial(8) (Rituximab for the Treatment of Wegener's Granulomatosis and Microscopic Polyangiitis)was a randomized, placebo-controlled, multicenter noninferiority trial that compared induction therapy with rituximab $\left(375 \mathrm{mg} / \mathrm{m}^{2}\right.$ per week for four weeks) or with oral cyclophosphamide ( $2 \mathrm{mg} / \mathrm{kg}$ per day) in 197 patients with GPA (75 percent of enrolled patients) or MPA (25 percent); Rituximab was noninferior to cyclophosphamide in inducing remission by six months (64 versus 53 percent). However, in the 100 patients with relapsing disease, rituximab was superior to cyclophosphamide in inducing remission (67 versus 42 percent) at six months. In the second trial (RITUXVAS) ${ }^{(9)}$, 44 patients with newly diagnosed antineutrophil cytoplasmic autoantibody (ANCA)-associated renal vasculitis were assigned in a 3:1 ratio to receive intravenous methylprednisolone (1000 $\mathrm{mg}$ ) followed by oral methylprednisolone plus either rituximab $\left(375 \mathrm{mg} / \mathrm{m}^{2}\right.$ per week for four weeks) or intravenous cyclophosphamide $(15 \mathrm{mg} / \mathrm{kg}$ every two weeks for three doses followed by infusions every three weeks). At 12 and 24 months in RITUXVAS, there was no difference in the rate of sustained remission (defined as the absence of disease activity for at least six months) between the rituximab- and cyclophosphamide-only groups (76 versus 82 percent). There was also no difference between groups in the rate of adverse events at 12 months. Maintenance therapy can be with tapering doses of steroids, azathioprine, methotrexate or rituximab. Close monitoring for extended period is mandatory as late relapses are well documented.Older age ( $>50$ years), impaired renal function (elevated creatinine levels), pulmonary manifestations at presentation with absence of ENT involvement are associated with an adverse outcome and increased mortality. ${ }^{(10)}$

\section{Summary}

The case reports summarises a young lady with pyrexia of unknown cause and left upper lobe consolidation who was diagnosed to have GPA based on appropriate serological testing and lung biopsy. Remission was achieved with steroids and rituximab and maintenance of remission is achieved by tapering doses of prednisolone.

Financial support and sponsorship - Nil No conflicts of interest

\section{References}

1. Godman GC, Churg J. Wegener's granulomatosis: pathology and review of the literature. AMA Arch Pathol. 1954 Dec;58(6):533-53. 
2. Falk RJ, Gross WL, Guillevin L, Hoffman GS, Jayne DRW, Jennette JC, et al. Granulomatosis with Polyangiitis (Wegener's): An alternative name for Wegener's Granulomatosis. Arthritis \& Rheumatism. 2011 Apr 1;63(4):863-4.

3. Lee KS, Kim TS, Fujimoto K, Moriya H, Watanabe H, Tateishi U, et al. Thoracic manifestation of Wegener's granulomatosis: CT findings in 30 patients. Eur Radiol. 2003 Jan;13(1):43-51.

4. Voswinkel J, Müller A, Lamprecht P. Is PR3-ANCA Formation Initiated in Wegener's Granulomatosis Lesions? Granulomas as Potential Lymphoid Tissue Maintaining Autoantibody Production. Annals of the New York Academy of Sciences. 2005 Jun 1;1051(1):12-9.

5. Mohammad AJ, Mortensen KH, Babar J, Smith R, Jones RB, Nakagomi D, et al. Pulmonary Involvement in Antineutrophil Cytoplasmic Antibodies (ANCA)associated Vasculitis: The Influence of ANCA Subtype. J Rheumatol. 2017 Oct;44(10):1458-67.

6. Stone JH, Hoffman GS, Merkel PA, Min Y-I, Uhlfelder ML, Hellmann DB, et al. A disease-specific activity index for Wegener's granulomatosis: Modification of the Birmingham Vasculitis Activity Score. Arthritis \& Rheumatism. 2001 Apr 1;44(4):912-20.

7. Yates M, Watts RA, Bajema IM, Cid MC, Crestani B, Hauser T, et al. EULAR/ERAEDTA recommendations for the management of ANCA-associated vasculitis. Annals of the Rheumatic Diseases. 2016 Sep 1;75(9):1583-94.

8. Stone JH, Merkel PA, Spiera R, Seo P, Langford CA, Hoffman GS, et al. Rituximab versus Cyclophosphamide for ANCA-Associated Vasculitis. New England Journal of Medicine. $2010 \mathrm{Jul}$ 15;363(3):221-32.
9. Jones RB, Cohen Tervaert JW, Hauser T, Luqmani R, Morgan MD, Peh CA, et al. Rituximab versus Cyclophosphamide in ANCA-Associated Renal Vasculitis [Internet]. http://dx.doi.org/10.1056/NEJMoa090916 9. 2010 [cited 2018 Nov 9]. Available from:

https://www.nejm.org/doi/10.1056/NEJMo a0909169?url_ver=Z39.88-2003\& rfr_id=ori\%3Arid\%3Acrossref.org\&rfr_da $\mathrm{t}=\mathrm{cr} \_$pub\%3Dwww.ncbi.nlm.nih.gov

10. Takala JH, Kautiainen H, Leirisalo-Repo M. Survival of patients with Wegener's granulomatosis diagnosed in Finland in 1981-2000. Scand J Rheumatol. 2010;39(1):71-6. 